\title{
Fabry-Perot spectroscopy: a powerful method for detecting superbubbles in galaxy discs
}

\author{
A. Camps-Fariña ${ }^{1,2}$, J. Beckman ${ }^{1,2,3}$, J. Zaragoza-Cardiel ${ }^{1,2}$, J. Font ${ }^{1,2}$ \\ and K. Fathi ${ }^{4}$ \\ ${ }^{1}$ Instituto de Astrofísica de Canarias, La Laguna, Spain \\ ${ }^{2}$ Departamento de Astrofísica, Universidad de la Laguna, Spain \\ ${ }^{3}$ Consejo Superior de Investigaciones Científicas, Spain \\ ${ }^{4}$ Department of Astronomy, Stockholm University, Stockholm, Sweden
}

\begin{abstract}
We present a new method for the detection and characterization of large scale expansion in galaxy discs based on $\mathrm{H} \alpha$ Fabry-Perot spectroscopy, taking advantage of the high spatial and velocity resolution of our instrument $(\mathrm{GH} \alpha \mathrm{FaS})$. The method analyses multi-peaked emission line profiles to find expansion along the line of sight on a pixel-by-pixel basis. At this stage we have centred our attention on the large scale structures of expansive gas which show a coherent gradient of velocities from their centres as a result of both bubble shape and projection effect. The results show a wide range of expansion velocities in these superbubbles, ranging from $30-150 \mathrm{~km} / \mathrm{s}$, with the expected trend of finding the higher velocities in the more violent areas of the galaxies. We have applied the technique to the Antennae and M83, obtaining spectacular results, and used these to investigate to what extent kinematically derived ages can be found and used to characterize the ages of their massive star clusters.
\end{abstract}

Keywords. galaxies: superbubbles, galaxies: kinematics, galaxies: evolution

$\mathrm{GH} \alpha \mathrm{FaS}$ is a Fabry-Perot integral field spectrometer, producing a 3.4'x3.4' data cube with a seeing-limited spatial resolution, and velocity resolution of $\sim 7-8 \mathrm{~km} / \mathrm{s}$. This allows us to separate different kinematic components of the line profile (normally $\mathrm{H} \alpha$ ) with the aim of detecting expansion.

In order to make this a systematic, galaxy-wide analysis, we need to automate the process of finding and fitting multiple components to the $\mathrm{H} \alpha$ profile. To do this we take advantage of the properties of the second derivative of a Gaussian, which tell us that for a combination of Gaussians each contribution lies between two zeros of the second derivative (Goshtasby \& O'Neill 1994). The position of the zeros is also related to the offset and width of each Gaussian, so we can estimate the parameters before fitting, to ensure an efficient solution.

We perform this on the entire cube, first detecting the number of components that can be separated in each profile and then fitting them to produce their final parameters. We then search for the expansion signature: two smaller peaks situated symmetrically at each side of the main, brightest peak. We produce new maps by assigning to each pixel the mean expansion velocity (distance from the main peak) and the mean intensity of the secondary peaks. In the poster Kinematic properties of superbubbles in the Antennae, M83 and Arp 270 we present results obtained aplying this method.

\section{References}

Goshtasby, A. \& O’Neill W., 1994, Graph. Models Image Process., 56, 281 